\title{
Introduction: The 5th Hellenic Conference on the History, Philosophy and Science Teaching "The Great Scientific Theories in the Teaching of Natural Sciences"
}

\author{
Konstantinos Korfiatis • Athanasios Raftopoulos • Demetris Portides
}

Published online: 28 January 2011

(C) Springer Science+Business Media B.V. 2011

Research on the contribution that the history and philosophy of science can make to the teaching of science has established itself as a dynamic scientific field. The international community of researchers continues to expand, publications on the subject are steadily increasing in number, and Science \& Education journal continually increases its impact and reputation. In Greece, since 2001, a community of researchers and teachers has been created and has organized successive Greek conferences on History, Philosophy and Science Teaching. The conference, from which papers were selected for this special issue, was the 5th conference. It, like the previous conferences, had as its basic target to promote an in-depth examination of the interplay between the history and the philosophy of science and the teaching of science.

The 5th Conference on History, Philosophy and Science Teaching was held in Nicosia, Cyprus, between 11 and 14 of June 2009. The organizing committee intended to broaden the character of the conference by inviting not only Greek speaking scientists and researchers but also scientists and researchers from all over the world to participate. A first step towards this direction was the invitation to distinguished foreign scientists to present their work, as well as the organization of an English-speaking symposium.

The theme of the 5th Conference was "The Great Scientific Theories in the Teaching of the Natural Sciences". The conference sought to reveal the interaction between the great scientific theories and science teaching, and especially the interaction between the Darwinian theory and science teaching highlighting questions concerning the impact of Darwinian theory for our general conception of scientific theories and for science teaching. An example is the extent to which contemporary discussions concerning the universality of the scientific laws, the limits of their application, and the type of explanation of the scientific

K. Korfiatis $(\bowtie)$

Department of Education, University of Cyprus, Nicosia, Cyprus

e-mail: korfiati@ucy.ac.cy

\author{
A. Raftopoulos \\ Department of Psychology, University of Cyprus, Nicosia, Cyprus \\ D. Portides \\ Department of Classics and Philosophy, University of Cyprus, Nicosia, Cyprus
}


phenomena they provide, have been influenced by the structure of Darwinian theory. Another example is the teaching of the scientific theories as a medium for explanation and prediction of phenomena which are characterized by limited fields of application and are continuously being criticized and revised.

Papers presented at the conference covered a wide range of issues, from Darwinism and quantum physics to socio-scientific issues and classroom case studies. In total 55 papers were presented. The five papers that appear in this volume (situated in relatively different disciplines ranging from Physics to Biology), explore directly or indirectly and from different perspectives the role of the Nature of Science (NOS) in science teaching. Two of the papers (Paraskeuopoulou and Koliopoulos; Constantinou and Papadouris) discuss the role of NOS directly by addressing the problem of the way students' understanding of NOS affects science teaching. In the same vein, Constantinou and Hatzilouka propose the development of a teaching innovation for the Nature of Science (NOS), which focuses on the interrelationship between science and technology, and which aims to bring to the fore the close and inextricable relationship between the two domains in modern scientific practice. Andreou's and Raftopoulos' paper approaches the problem indirectly by elaborating on the differences and similarities between the views of scientists from the ancient Greeks up to the late Arabic tradition on the nature of light and sight, on the one hand, and the views of high-school students on the same subject, on the other. NOS, and more specifically the underpinnings of the scientific enterprise, may explain both similarities and differences between the scientists' and students' activities. Finally, Hovardas and Korfiatis explore the "Balance of Nature" metaphor in Biology and Environmental Education, which is one of the major paradigms in the field, and its recent scientific standing and suggest that the exclusive teaching of the "Balance of Nature" metaphor produces cultural, scientific and learning misconceptions about the structure and function of nature. NOS is involved in the sense that the way scientific theories develop and change, one of the critical elements of NOS, should make instructors and curricula designers sensitive to the fact that theories have their limitations and, consequently, change, even within the same paradigm.

Paraskeuopoulou and Koliopoulos present the results of a research project that investigates the extent to which the use of the historical episode of the Millikan-Ehrenhaft dispute over the existence of the elementary electric charge can improve students' conceptions of specific aspects of NOS. These aspects are: (1) The role played by empirical data in scientific debate; (2) the distinction between observation and inference; (3) the role of the scientist's imagination and creativity in the formation of theory in the natural sciences, and (4) the fact that the natural sciences have a subjective content during the formation of a theory.

They presented results of a teaching program containing seven hourly teaching units was designed and implemented. The teaching intervention was designed on the basis of an explicit form of teaching that was integrated into the scientific content and through the use of short stories. Students' conceptions of specific aspects of NOS were documented in a questionnaire distributed before and after the class. The results showed that there was a significant statistical improvement in students' conceptions of the aspects of NOS that had been selected for teaching. More specifically, there was an improvement in the conceptions of many students after the teaching intervention in all four aspects of NOS covered in the study. There was a statistically significant improvement in the students' conceptions for each of the four aspects of NOS. Although prior to the teaching intervention no student had exhibited 'informed' conceptions of all four of the NOS aspects under investigation, after the teaching intervention two students exhibited 'informed' conceptions of all four of the 
NOS aspects. Moreover, none of the remaining students exhibited only 'simple' conceptions.

Constantinou and Papadouris address the problem of learning about energy, which is recognized as an important objective of science teaching starting from the elementary school. This creates the need for teaching simplifications that compromise the abstract nature of this concept with students' need for a satisfactory qualitative definition. Conventional teaching approaches have failed to respond to this need in a productive manner. In an attempt to maintain consistency with how energy is understood in physics, they tend to either provide abstract definitions or bypass the question "what is energy?" which is vitally important to students. In their paper, the authors describe the epistemological barriers that are inherent in conventional attempts to introduce energy as a physical quantity and suggest that shifting the discussion to a philosophically-oriented context could provide a means to address them in a productive manner. They propose a teaching approach, for students (age range 11-14), that introduces energy as an entity in a theoretical framework that is invented and gradually elaborated in an attempt to analyze the behavior of diverse physical systems, and the various changes they undergo, using a coherent perspective.

The authors propose that this theoretical framework provides an epistemologically appropriate context that lends meaning to energy and its various features. They argue that this philosophically informed teaching transformation provides a possible means to overcome the various shortcomings that typically characterize attempts to introduce and elaborate the construct of energy while at the same time it allows integrating, in a meaningful and coherent manner, learning objectives relevant to the understanding of the Nature of Science (NOS), which is recognized as a valuable component of learning in science.

Contantinou and Hatzilouka's paper examines the development of a teaching innovation for the nature of science (NOS), for students aged 11-14, which specifically focuses on the interrelationship between science and technology (S\&T). The development of the teaching and learning materials relied on inputs from three sources: the history and philosophy of science and technology, existing knowledge concerning the teaching and learning about the NOS, empirical data on students' initial ideas and difficulties about this topic. The first served to provide an account for the various forms of interaction between science and technology, which, in turn, guided the formulation of epistemologically coherent learning objectives. The second provided the pedagogical grounds on which to base the design of the activities. The third facilitated the design of activities that build on students' productive initial ideas, while providing them with guidance to resolve the difficulties they tend to encounter.

The authors maintain that the paper's main contribution is twofold. First, it highlights the potential of using inputs from the history and philosophy of science on perspectives of the interrelationship between science and technology. This serves as a useful tool for facilitating and supporting attempts to develop teaching and learning materials that address NOS issues. At the same time, the lessons learned from mapping the historical background around the certain perspectives of the interrelationship between $S \& T$ serve to articulate the teaching transformation, by identifying the constraints that is bounded with and ensuring its potential to be elaborated at subsequent stages so as to address these constraints and become more closely aligned with how $S \& T$ are construed in the corresponding academic fields.

The second contribution of the paper relates to the proposal of the structure of a teaching innovation for addressing this issue with elementary students. Despite the wide 
recognition of the importance of helping students develop epistemologically informed views about the NOS, this learning objective typically receives scant attention in conventional teaching practice in science and there is a need for relevant research-based teaching innovations for integrating explicit aspects of the NOS, especially in the case of the elementary and middle school grades. The paper contributes towards meeting this need by proposing an activity sequence for developing students' awareness of a specific NOS aspect, namely the interrelationship between $S \& T$.

Andreou and Raftopoulos start from the assumption that there are two indisputable findings in science education research. First, students go to school with some intuitive beliefs about the natural world and physical phenomena that pose an obstacle to the learning of formal science. Second, these beliefs result from the confluence of two factors, namely, their everyday experience as they interact with the world around them and a set of operational constraints or principles that channel both perceptually and conceptually the way these experiences are perceived and interpreted. History of science suggests that the theories of early scientists through which they sought to explain physical phenomena relied mostly on ideas that closely fitted their experiences of the relevant phenomena. This characteristic of the early scientific ideas is the root of the epistemological difficulties that early scientists faced in their attempts to explain the phenomena. The authors focus on the early theories in optics (from ancient Greek to the late Islamic scientific traditions) and argue that students face some of the same epistemological problems as early scientists in explaining vision and optical phenomena for the reason that students' intuitive beliefs are also closely tied to particular phenomena and as a result the underlying notions are fragmentary and lack the necessary generality that would allow them to cover many disparate phenomena. Knowledge of these epistemological problems can help the instructor to identify the key elements for a better understanding of the formal theory of optics and, in turn, lead to a more effective instruction.

The historical background and the documented misconceptions of the students show that students and early scientists face to some extent the same epistemological obstacles that arise from experience and their intuitive beliefs. Three such obstacles are identified, to wit, the conceptualization of the ray, the model for the emission of radiation, and the psychology of vision. The experience from the history of science yields sufficient evidence that these three concepts provide the necessary framework to properly explain a wide range of phenomena of everyday life. Understanding these concepts may lead to more effective teaching in geometrical optics and allow teachers to introduce, at a later stage, new tools or models that describe the nature of light as it is manifested in other more complex phenomena.

Finally, Hovardas and Korfiatis' paper discusses the "Balance of Nature" metaphor, which is a pervasive idea in ecology. Recently, the scientific community has acknowledged that equilibrium conditions are rare, while disturbance events are not uncommon. In view of this, the authors suggest that teaching exclusively the "Balance of Nature" metaphor produces cultural, scientific and learning misconceptions about the structure and function of nature. The paper outlines an exemplary educational intervention for high school students to show that the use of computer simulations could serve important educational goals in ecology and environmental education, such as the emancipation of the concept of "balance" from its metaphysic burden, the comprehension of the dynamics and the systemic nature of ecological processes and the appreciation of the mutual relation between society and nature.

Teaching ecology requires a pluralistic and complete picture of the important traits of the discipline such as the scientific method(s) that ecology implements with rigor and 
devotion. Ecology has developed from what was once regarded as 'natural history' into a science with a rich array of theories, concepts and models and this image should be embodied in educational processes. Addressing patterns of change in educational theory and practice would better satisfy the scope of science and environmental education. However, attempts to implement in educational practice a science-critical approach in ecology have been extremely limited. Especially for environmental education, the reluctance of the field to accept a critical re-appraisal and teaching of ecology renders itself inconsistent with its own declarations concerning criticism of modern science. To cover this gap, the proposed intervention focuses on the paradigm of population ecology and presents a critical revisit of the "Balance of Nature" metaphor.

The authors argue that messages promoted through environmental education should focus on the interplay between society and nature. The authors also suggest that environmental education programs should not be confined to mere descriptions of biodiversity; instead, they should address the compatibility of certain types of human interventions in concrete spatial and temporal contexts with environmental conservation. For instance, ecotourism development could compensate locals for income lost due to the implementation of environmental management measures, provided that natural and social carrying capacities are not overridden. Moreover, since the decline of primary sector activities might allow forests to expand over open spaces and lead to reduced habitat heterogeneity and biodiversity indices, park managers might support grazing, logging, and agricultural practices in order to create and maintain clearings in the forest. The ability to discuss these types of synergies is crucial for developing critical thinking to support environmental literacy and create a politically informed public able to handle complex environmental issues.

This is the fourth time that Science \& Education has published special issues based on reviewed papers that were presented in Hellenic conferences on the History, Philosophy and Science Teaching (for previous special issues, see Skordoulis and Halkia 2005; Skordoulis 2008; Koliopoulos 2009). We would like to give our warmest thanks to Michael R. Matthews, the editor of Science \& Education, for giving the opportunity to present selected papers to an international readership.

The next conference will be held in Thessaloniki, Greece, 1-5 July 2011, in a joint venture with the 11th International Conference on History, Philosophy and Science Teaching, having a special focus on the interrelations of science and culture. In this context the local community of Greek researchers on history, philosophy and science teaching will join its forces with the international community in order to create a promising forum for discussion and reflection on the contribution of history and philosophy of science in science teaching.

\section{References}

Koliopoulos, D. (2009). The 4th Hellenic conference on the history and philosophy of science and science teaching "the cultural component of science in education": Introduction. Science \& Education, 18, 1101-1103.

Skordoulis, C. (2008). Hellenic studies in history, philosophy of science and science teaching: new perspectives. Science \& Education, 17, 1193-1197.

Skordoulis, C., \& Halkia, J. (2005). Introduction: Notes on the development of history, philosophy and science teaching in Greece. Science \& Education, 14, 7-8. (Special issue: selected proceedings from the 2nd Hellenic HPST conference). 